\title{
Gamma 3 Long Nail for Complex Sub-Trochanteric Fractures: A Ten-Year Retrospective Study
}

\author{
Urso R ${ }^{1}$, Milani L' ${ }^{1 *}$, Ortolani A $^{1}$, Martucci A', \\ Berti M1, Pascarella $R^{2}$ and Tigani $D^{1}$ \\ ${ }^{1}$ Department of Trauma and Orthopaedic Surgery, \\ Ospedale Maggiore Carlo Alberto Pizzardi, Bologna, Italy \\ ${ }^{2}$ Department of Trauma and Orthopaedic Surgery, \\ Ospedali Riuniti di Ancona, Ancona, Italy \\ *Corresponding author: Milani L, Department of \\ Trauma and Orthopaedic Surgery, Ospedale Maggiore \\ Carlo Alberto Pizzardi, Largo Nigrisoli 2, 40133 Bologna, \\ Italy
}

Received: September 20, 2021; Accepted: October 19, 2021; Published: October 26, 2021

\begin{abstract}
Cephalomedullary nailing is considered the treatment of choice for trochanteric and subtrochanteric femoral fractures. The aim of this study was to report postoperative outcomes of one of the widely used trochanteric nail device, the Gamma 3 long nail. We retrospectively assessed 405 patients treated with Gamma 3 long nail in a single Level One Trauma Centre between 2010 and 2018. We finally included 261 ambulant patients with 65 years or older, a lowenergy trauma and a closed trochanteric or subtrochanteric femoral fracture. Clinical outcomes were evaluated using the Hip Fracture Functional Recovery Score (FRS), while radiological complications and failures were assessed on postoperative $\mathrm{x}$-rays.
\end{abstract}

More than two-thirds of patients had completed fracture consolidation within 4 months after surgery. Immediate full weight bearing was allowed postoperatively in $64.0 \%$ of patients. We reported a mortality of $24.5 \%$ at one year postoperatively. The leading clinical postoperative complication was anemia (69.3\%), followed by deep venous thrombosis (7.7\%). Coxa vara was observed in 73 patients $(28 \%)$, followed by malreduction in flexion-external rotation of the proximal femoral fragment $(26.8 \%)$. No case of lag screw cutout was reported. Our study indicated that last generation of Long Gamma nail is a reliable implant for trochanteric and subtrochanteric femoral fractures in the elderly patients, leading to high rate of bone union and reduced incidence of related complications. An excellent fracture reduction and prevention of postoperative varus malalignment are the main factors that can avoid the major postoperative complications and failures after Gamma 3 long nailing.

Keywords: Gamma 3 long nailing; Subtrochanteric femoral fractures; Complex trochanteric fractures; Complications; Failures; Clinical outcomes

\section{Ablbreviations}

LGN: Long Gamma Nail; FRS: Hip Fracture Functional Recovery Score; ROC: Radius of Curvature; ASA: American Society of Anesthesiologists; BADL: Basic Activity of Daily Living; IADL: Instrumental Activity of Daily Living; Max: Maximum; N.: Number; ext.: External-Rotation; fem.: Femoral

\section{Introduction}

Gamma nail was developed for the treatment of trochanteric hip fractures in the mid 1980's and was first brought into clinical use in 1988 [1].

Long Gamma Nail (LGN) was introduced some years later in 1992 for the treatment of subtrochanteric fractures, femoral shaft fractures and combined trochantero-diaphyseal fractures of the femur. The later implant has experienced further changes in 1997, 2003 and 2018 [2].

The second generation of LGN was introduced in 1997 with reduced medio-lateral curvature from $10^{\circ}$ to $4^{\circ}$ and modifications of proximal and distal caliber in a single diameter of 17 and $11 \mathrm{~mm}$ respectively [3]. In 2003 appeared the long Gamma 3 nail [2]. In contrast with the previous one, The Radius of Curvature (ROC) of the femoral nail was reduced to $2.0 \mathrm{~m}$ to match the femoral bowing.
Others change concerned the introduction of a titanium alloy with anodized surface treatment for the implant, the reduction of the proximal diameter to $1.5 \mathrm{~m}$, and the modification of both proximal lag screw and distal locking screws [4].

The purpose of this study was to report the treatment results of complex trochanteric and subtrochanteric femoral fractures in the elderly patients with last generation of Long Gamma nail and determine the rates and the time of consolidation, all implant-related complications and patient mortality.

\section{Materials and Methods}

A retrospective analysis of all patients treated with a Gamma 3 Long Nail (Stryker) was performed in a single Italian Trauma Centre within a ten-year period (between January 2009 and December 2018).

All procedures performed in studies involving human participants were in accordance with the ethical standards of the institutional and national research committee and with the 1964 Helsinki Declaration and its later amendments or comparable ethical standards. The study was approved by the Bioethics Committee "Area Vasta Emilia Centro" (CE-AVEC) of the Medical University of Bologna.

Informed consent was obtained from all individual participants included in the study. All patients signed informed consent regarding 
publishing their data and photographs.

At a first search we found 405 patients. The inclusion criteria for this study were patients 65 years of age or older with trochanteric (AO/ASIF 31-A), subtrochanteric (AO/ASIF 32-A) or combined trochantero-diaphyseal fractures (Table 1).

We included a total of 261 patients with a mean age of 84.8 years old (range 65 - 101).

$77.8 \%$ of patients were females $(n=203)$, while $22.2 \%$ of patients were males $(n=58)$. The lower limb surgically treated was the right one in 144 patients (55.2\%), while the left one in 117 patients (44.8\%).

According to the AO/ASIF classification, we found 199 trochanteric (AO 31-A) fractures (76.2\%), 54 subtrochanteric (AO 32-A) fractures $(20.7 \%)$ and eight combined trochantero-diaphyseal (AO 31-A + AO 32-A/B/C) fractures (3.1\%). Details are reported in (Table 1).

The mean preoperative ASA score was 3.3 (range 2 - 4), while the mean preoperative CCI was 1.8 (range 0 - 10).

The patients were operated on a traction table in a supine position according to standard technique using an image intensifier. General anesthesia was used in only 27 cases, whereas spinal anesthesia was received in 234 cases.

Closed reduction was achieved by adjusting the traction force, limb alignment and rotation. The intramedullary nail was then inserted routinely without any open procedure in 179 cases. In 82 patient it was necessary a clamp-assisted reduction technique through a mini-open access

Moreover, 154 patients (59\%) were surgically treated in the first 48 hours after injury. The mean time from recovery to surgery was 3.1 days (range 0 - 14), the mean operative time was 96 minutes (range 30 - 180), while the mean hospital stay was seven days (range 2 - 27).

One hundred and eighty-one patients (69.3\%) needed blood transfusions after surgery. In particular, the mean postoperative number of blood transfusions was 1.9 (range 0 - 9).

According to previous papers [5-7] fracture union was defined as follows. Clinically, absence of pain and no tenderness at the site of fracture with possibility by the patient to walk without aids; radiographically, solid callus with cortical density had bridged the fracture fragments. Delayed union was defined as persistent pain and no sign of bridging callus after four months postoperatively. Nonunion was defined as persistent pain and no sign of bridging callus six months postoperatively [1].

164 patients were submitted to immediate weight bearing whereas 97 patients who had more than $10^{\circ}$ of residual varus/valgus misalignment on the anteroposterior and lateral radiographs the weight bearing was delayed. In 39 cases it was allowed after four weeks, in 39 cases after eight weeks, in 12 cases after 12 weeks, and in three cases after 16 weeks. Once discharged, 170 patients (65.1\%) went to a rehabilitation institute, while 83 patients $(31.8 \%)$ returned to home.

Quality of fracture reduction was assessed on postoperative radiographs. We considered the reduction unsatisfactory in case of misalignment on the anteroposterior radiograph of more than $10 \mathrm{~mm}$ of any fragment, $10^{\circ}$ of varus/valgus angulation, and/or more than $10^{\circ}$ of angulation on the lateral radiograph.

Additionally, we evaluated the entry point of the intramedullary nail on the greater trochanter and the position of the lag screw into the femoral neck to verify if they can influence the postoperative rate of failures and radiological complications. We considered the femoral neck dividing it into 9 regions (postero-inferior, postero-central, postero-superior, central-inferior, central, central-superior, anteroinferior, antero-central, and antero-superior) [4].

All patients were contacted by phone for investigate the clinical outcomes (preoperative, at one month and at one year postoperatively) using the Hip Fracture Functional Recovery Score (FRS) [8]. This questionnaire includes 11 items: four about BADL (bathing, feeding, dressing, toileting), six about IADL (food shopping, housework, laundry, food preparation, banking/finances, use of transportation) and one about mobility (walk outdoors/indoors). After this screening, we had 185 patients with a clinical follow-up and 168 patients with a radiological follow-up superior of six months.

The mean clinical follow-up of the living patients was 49.5 months (range 14 - 128), while the mean radiographic follow-up was 17.9 months (range 12 - 103).

All statistical analysis was performed using MedCalc statistical software (version 19.5.1) in consultation with a statistician. Data were analysed using Chi-Square test and Fischer's Exact test, and were presented as mean, Standard Deviation (SD) and range. Statistical differences were reported as $\mathrm{p}<0.05$. The survival study with related variables was performed using the Cox-regression model.

\section{Results}

A complete consolidation of the fracture within four months after surgery was observed in 199 patients with a rate of $76.2 \%$. On the contrary 56 patients $(21.5 \%)$ had a consolidation within months (delayed union), and only six patients (2.3\%) with fracture non-union. 167 patients of 199 who obtained adequate timing of consolidation had been submitted to immediate weight bearing protocol, whereas 62 patients of 94 who had delayed load on the affected limb belong to the group of delayed union or fracture non-union.

Four intraoperative additional femoral fractures (1.5\%) had been reported: in three cases they did not modify the prognosis, while in one case the weight bearing was delayed to three months postoperatively.

At the radiological analysis no case of postoperative lag screw migration or cut-out was noted.

The main clinical and radiological postoperative complications were reported in (Table 2). Those include 72 patients (27.6\%) with postoperative varus deformity, exceeding the limit of ten degrees of the operated hip compared to the contralateral limb, 70 patients (26.8\%) with a malredution in valgus-external rotation of the proximal femoral fragment, 65 patients $(24.9 \%)$ with a malreduction in flexion of the proximal femoral fragment, and 49 patients (18.8\%) had an entry point of the intramedullary nail too lateral to the apex of the greater trochanter. 


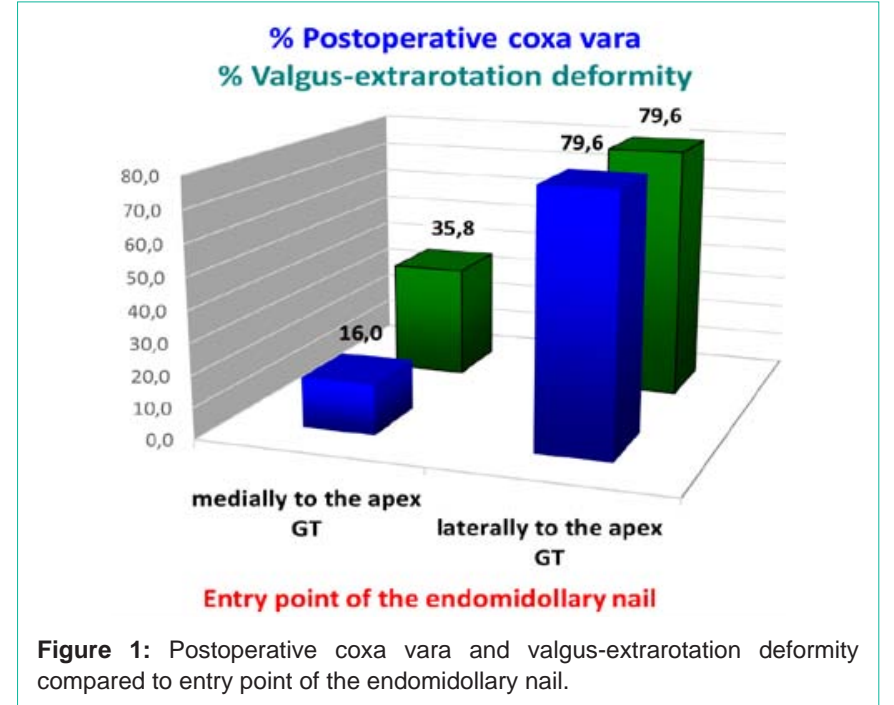

We found $79.6 \%$ of patients had a postoperative coxa vara when the entry point was too lateral to the apex of the greater trochanter, while only $16.0 \%$ of patients had this deformity when the entry point was correct, medially at the apex of the greater trochanter $(\mathrm{p}<0.001)$ (Figure 1). Moreover, we found $79.6 \%$ of patients had a valgusexternal rotation deformity of the proximal fragment when the entry point of the intramedullary nail was too lateral to the apex of the greater trochanter, while $35.8 \%$ had this deformity when the entry point was correct $(\mathrm{p}<0.001)$ (Figure 1).

Failures were reported in two cases $(0.8 \%)$ and needed revision surgery: the first patient due to a significant malreduction of the fracture with 18 degrees of varus angulation, and the second one due to rupture of the nail with concomitant non-union at ten months postoperatively. In both cases it was performed an intramedullary nail removal, a new internal reduction by a direct lateral approach, and an osteosynthesis with a femoral locking compression plate.

Additionally, comparing postoperative load with the presence or absence of valgus-external rotation deformity of the proximal fragment on postoperative x-rays, we found this deformity occurred in $29.3 \%$ of patients (49 out of 167) with immediate full weight bearing, in $61.5 \%$ of patients (24 out of 39 ) with delayed weight bearing to one month, while in $76.4 \%$ of patients ( 42 out of 55 ) with delayed weight bearing to two month and over $(\mathrm{p}<0.001)$. In particular, this deformity occurred in $76.8 \%$ of patients (30 out of 39 ) with delayed weight bearing to two months, while in $75 \%$ of patients (9 out of 12, 3 out of 4) with delayed weight bearing to three and four months respectively (Figure 2).

Regarding the position of the lag screw into the femoral neck, we found a postero-inferior position in 83 cases $(31.8 \%)$, a posterocentral position in 74 cases $(28.4 \%)$, a central-central position in 50 cases (9.5\%), a central-inferior position in 23 cases (8.8\%), a posterosuperior position in 16 cases (6.1\%), a central-superior position in six cases $(2.4 \%)$, an antero-inferior position in three cases $(1.1 \%)$, an antero-central position in three cases (1.1\%), an antero-superior position in two cases $(0.8 \%)$. No cases of cut-out have been reported.

As for the clinical outcomes, the mean preoperative total FRS was 28.6 (range 13 - 44), the mean postoperative FRS at one month was

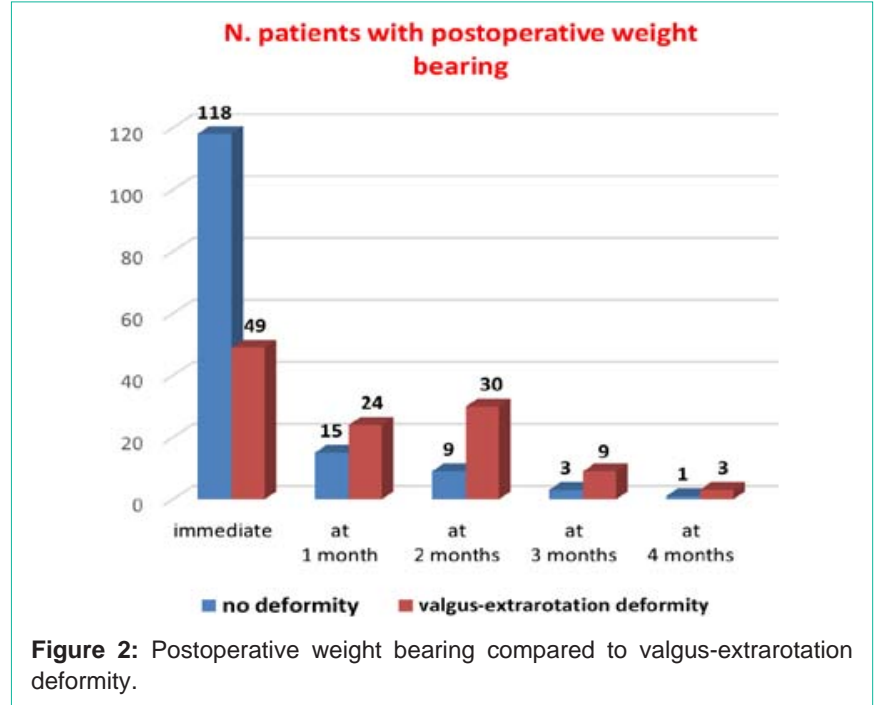
deformity.

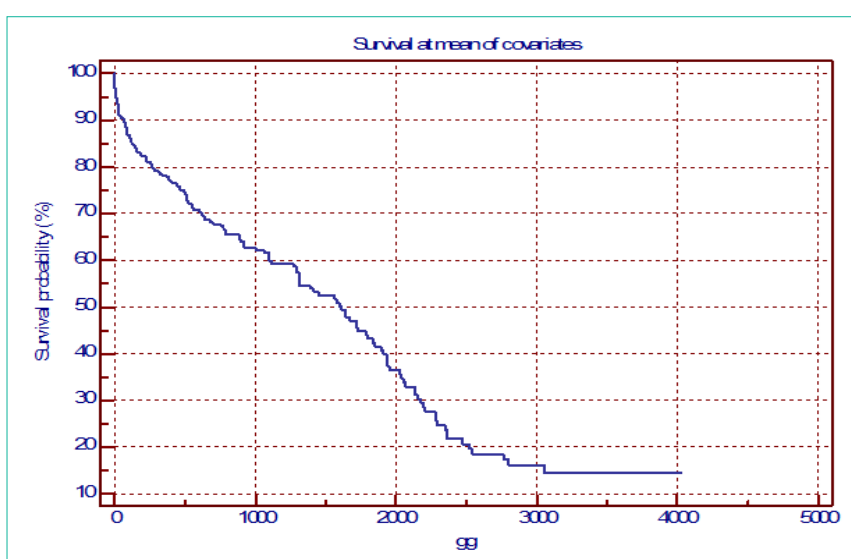

Figure 3: Kaplan - Meier survival curve.

Table 1: Type of AO/ASIF fractures included.

\begin{tabular}{|l|c|}
\hline \multicolumn{1}{|c|}{ Fractures included } & N. (\%) \\
\hline AOIASIF 31-A & $199(76.2 \%)$ \\
\hline $31-A 1$ & $2(0.8 \%)$ \\
\hline $31-A 2$ & $2(0.8 \%)$ \\
\hline $31-A 3$ & $178(68.1 \%)$ \\
\hline $31-A 1+A 3$ or 31-A2+A3 & $17(6.5 \%)$ \\
\hline AOIASIF 32-A & $54(20.7 \%)$ \\
\hline AOIASIF 31-A + 32A/B/C combined & $8(3.1 \%)$ \\
\hline
\end{tabular}

19.8 (range 9 - 39), while the mean postoperative FRS at one year was 27.7 (range 12 - 44). Details about the subgroup data of BADL, IADL and mobility are reported in (Table 3 ).

The post-operative mortality reached the value of $9.6 \%(25$ patients of 261) at one month eight patients (3.1\%) died during hospital stay. At one year the mortality rate was $24.5 \%$ (64 patients of 261). No patient died during the surgical procedure. In patients died within one month postoperatively the mean preoperative CCI was 2.4, while in patients died within one year postoperatively it was 2.7. Furthermore, we analysed the probability of patients' survival surgically treated with gamma long nail using the Cox regression 
Table 2: Clinical and radiological postoperative complications.

\begin{tabular}{|l|l|}
\hline \multicolumn{1}{|c|}{ Clinical complications, N. (\%) } & \multicolumn{1}{c|}{ Radiological complications, N. (\%) } \\
\hline Anemia, 181 (69.3\%) & No complications, 93 (57.8\%) \\
\hline Deep vein thrombosis, 20 (7.7\%) & Coxa vara, 72 (27.6\%) \\
\hline Cardiac failure, 13 (5.0\%) & Malreduction valgus-ext, 70 (26.8\%) \\
\hline Pleural effusion, 10 (3.8\%) & Malreduction flexion, 65 (24.9\%) \\
\hline Pneumonia, 9 (3.4\%) & Delayed union, 56 (21.5\%) \\
\hline Acute renal failure, 5 (1.9\%) & $\begin{array}{l}\text { Lag screw protrusion in soft tissue, 25 } \\
(9.6 \%)\end{array}$ \\
\hline Superficial wound infection, 5 (1.9\%) & $\begin{array}{l}\text { Impingement anterior fem. cortex, 25 } \\
(9.6 \%)\end{array}$ \\
\hline Ictus cerebri, 4 (1.5\%) & Breakage of distal screws, 10 (3.8\%) \\
\hline Respiratory failure, 3 (1.1\%) & Intraoperative femoral fractures 4 (1.5\%) \\
\hline Pulmonary embolism, 2 (0.8\%) & Non-union, 6 (2.3\%) \\
\hline Septic shock, 1 (0.4\%) & Mobilization of distal screws, $2(0.8 \%)$ \\
\hline
\end{tabular}

Table 3: Hip Fracture Functional Recovery Score (FRS) results.

\begin{tabular}{|c|c|c|}
\hline $\begin{array}{c}\text { Mean preoperative } \\
\text { FRS } \\
\text { (max. value) }\end{array}$ & $\begin{array}{c}\text { Mean postoperative } \\
\text { FRS } \\
\text { at } 1 \text { month (max. value) }\end{array}$ & $\begin{array}{c}\text { Mean postoperative } \\
\text { FRS } \\
\text { at 1 year (max. value) }\end{array}$ \\
\hline Total FRS: $28.6(44)$ & Total FRS: $19.8(36)$ & Total FRS: $27.7(44)$ \\
\hline BADL: $10.9(16)$ & BADL: $7(14)$ & BADL: $13.2(16)$ \\
\hline IADL: $15.2(24)$ & IADL: $11.2(20)$ & IADL: $14.6(24)$ \\
\hline Mobility: $2.5(4)$ & Mobility: $1.4(4)$ & Mobility: $2.5(4)$ \\
\hline
\end{tabular}

model, considering a series of six covariates (age, gender, side, ASA, type of fracture, and time between fracture and surgery). The probability of survival was reported using the Kaplan - Meier curve (Figure 3).

We found that the one-year survival probability was $76 \%$, it was reduced to $62 \%$ at three years after surgery, to $45 \%$ at 5 years, and then there was a drastic reduction of survival to $27 \%$ at six years, to $17 \%$ at eight years, and up to $15 \%$ at ten years postoperatively. In particular, we noted age ( $p<0.0001)$, gender $(\mathrm{p}<0.0005)$, ASA $(\mathrm{p}<0.00284)$, and fracture side $(\mathrm{p}<0.0295)$ had a significative influence on the cumulative patients' survival, while the type of fracture according to the AO classification $(p=0.38)$ and the time between fracture and surgery $(p=0.31)$ did not significantly affect patients' survival. Of note, we found patients with ASA score of 2 had a mean survival time of 1132 days, while patients with ASA score of 3 and 4 had a mean survival time of 987 days and 662 days, respectively.

\section{Discussion}

Based on the results of the current study, we believe that long Gamma nail could be considered the treatment of choice for the treatment of trochanteric and subtrochanteric femoral fractures in elderly patients. Particularly, we obtained fracture consolidation in 255 of 261 patients, of which 199 (76.2\%) within four months. These data are in line with reported by other authors [9-11] that showed a mean bone healing time of four months (range 3 - 9 months), attesting the difficulty in the healing of these difficult fractures.

The long gamma nail joins the load sharing proprieties of an intramedullary nail with the sliding characteristic of the hip screw: this union consents a decreasing of $25-30 \%$ in the bending stress on the nail and a reduction of the implant failure rate in comparison with extramedullary implants $[12,13]$ improving the capacity to obtain a more rapid healing of the fractures in respect of the closed biological treatment of fractures [14]. The low incidence of failures with long gamma nail in our study (two cases, rate of $0.8 \%$ ) is in line with this principle: we found one intramedullary nail breakage and one not tolerable malreduction of the fracture which necessitated an early revision with a proximal femoral locking compression plate. Furthermore, postoperative mortality was found to be higher in older, female patients with a greater number of comorbidities. These situations are in line with the current literature and are due to the characteristics of these fragile patients [15]. In this cohort, the comorbidities of our patients were particularly important as the mean ASA score was 3.3, as well as the number of clinical general complications observed. The general complications rate, blood loss, duration of surgical procedure, length of hospital stay and mortality at 30 days $(9.6 \%)$ were comparable to other published series $[3,9,13,16]$, whereas we noted the one year mortality $(24.5 \%)$ was lower than the rates reported in the orthopedic literature $(28-38 \%)[13,17,18,19]$.

A great number of variables may predispose these fragile patients to general complications. Certainly, the enlarged anterior arch of femur normally presents in old patients with osteoporosis, as well as the distal locking, makes the insertion of long nails more difficult and thus elongate the operation time [17]. Moreover, long nail needs a longer distance of intramedullary reaming, which increases intraoperative bleeding and the need for transfusion [18]. Finally, Bjorgul et al. reported that higher ASA score could be a predictor of an increased risk of mortality after hip fracture surgery [19]. In line with that, in our study we found patients with ASA score of 4 had a mean time between surgery and death of 662 days, patients with ASA score of 3 had a mean time of 987 days, while patients with ASA score of 2 had a mean time of 1132 days.

Even if a certain number of complications have been reported in our series in regarding with the past, this implant has proven a significant reduction of rate of local complications and healing of the fracture site [9-11]. Particularly, we can observe a drastic reduction of intraoperative additional fractures in comparison with the first series $[4,13,18]$. Source of debate is the difficulty to obtain a sufficient reduction of these complex fractures. Several studies in literature highlighted the entry point of the intramedullary nail can influence the postoperative reduction of the fracture and the alignment of the affected lower limb $[11,14,19]$. These data have been confirmed in our study and we found an entry point too lateral to the apex of the greater trochanter can significantly increase the incidence of malreduction in valgus-external rotation and flexion of the proximal femoral fragment. Krappinger et al. [15] proved intraoperative correction of varus malalignment and improvement of the medial cortical support are very important to avoid non-union after gamma long nailing of subtrochanteric femoral fractures. Several reduction techniques can be used to overcome the deforming forces presents in the proximal femur to allow for proper nail placement. Short nail or specific tools to guide the K-wire placement is crucial because it provides the correct path for the reamers and the maintaining of fracture reduction. For the same purpose Schanz pins with T-handles have been proposed [20]. For comminuted or long spiroid fractures a temporary clamp inserted by a short open incision could be useful in many cases $[14,20]$. 
Several studies in literature [21-23] noted that the position of the lag screw in the central, infero-central or infero-posterior part of the femoral neck gave better functional results if compared with other positions. In our study we found 207 patients (79.3\%) with a central, infero-central or infero-posterior position of the lag screw in the femoral neck. Among these 207 patients, 81 (39.1\%) did not have any postoperative complications, while $39(18.8 \%)$ had a valgusexternal rotation deformity of the proximal fragment. Therefore, our data confirmed lag screw position in one of the three positions into the femoral neck (central, infero-central and infero-posterior) was associated with a lower rate of postoperative complications as coxa vara, valgus-external rotation deformity and malreduction in flexion of the proximal fragment.

Moreover, Shetty et al. [24] demonstrated the Radius of Curvature (ROC) of the long Gamma nail can influence the complications in the distal femoral shaft. They showed only a $18.5 \%$ of patients with a ROC of $1.5 \mathrm{~m}$ had the tip of the nail in anterior one-third of the canal compared to $80 \%$ of patients with a ROC of $2.0 \mathrm{~m}$. In our study 249 patients of 261 (95.4\%) were treated with a long 3 Gamma nail with a ROC of 2.0 while only 12 patients (4.6\%) with a ROC of 1.5 . In particular, we found 25 patients $(9.6 \%)$ with an impingement of the distal femoral anterior cortex: in 23 of these 25 cases (92\%) it was used a long Gamma nail of $2.0 \mathrm{~m}$.

\section{Conclusion}

The results of this study indicate that last generation of Long Gamma nail is a reliable implant for trochanteric and subtrochanteric femoral fractures in the elderly patients, leading to high rate of bone union and reduced incidence of related complications. However, it is very important to prevent postoperative Varus malalignment and obtain a good alignment in order to prevent significant angular and rotation deformity (particularly valgus-external rotation of the proximal fragment) as they are the main factors for postoperative complications. However, entry point of the intramedullary nail and positioning of the lag screw into the femoral neck are additional factors that can significantly affect on the postoperative reduction and alignment.

\section{References}

1. Bojan AJ, Beimel C, Speitling A, Taglang G, Ekholm C, Jonsson A. 3066 consecutive Gamma Nails. 12 years experience at a single centre. BMC Musculoskelet Disord. 2010; 11: 133.

2. Pascarella R, Fantasia R, Maresca A, Bettuzzi C, Amendola L, Violini S, et al How evolution of the nailing system improves results and reduces orthopedic complications: more than 2000 cases of trochanteric fractures treated with the Gamma Nail System. Musculoskelet Surg. 2016; 100: 1-8.

3. Georgiannos D, Lampridis V, Bisbinas I. Subtrochanteric femoral fractures treated with the Long Gamma $3^{\circledR}$ nail: A historical control case study versus Long trochanteric Gamma nail ${ }^{\circledR}$. Orthop Traumatol Surg Res. 2015; 101: 675 680 .

4. Barquet A, Francescoli L, Rienzi D, López L. Intertrochanteric-subtrochanteric fractures: treatment with the long Gamma nail. J Orthop Trauma. 2000; 14: 324-328.
5. Tigani D, Fravisini M, Stagni C, Pascarella R, Boriani S. Interlocking nail for femoral shaft fractures: is dynamization always necessary? Int Orthop. 2005; 29: 101-104.

6. Wu CC, Chen WJ. Healing of 56 segmental femoral shaft fractures after locked nailing. Poor results of dynamization. Acta Orthop Scand. 1997; 68: 537-540.

7. Tay WH, de Steiger R, Richardson M, Gruen R, Balogh ZJ. Health outcomes of delayed union and nonunion of femoral and tibial shaft fractures. Injury. 2014; 45: 1653-1658.

8. Zuckerman JD, Koval KJ, Aharonoff GB, Skovron ML. A functional recovery score for elderly hip fracture patients: II. Validity and reliability. J Orthop Trauma. 2000; 14: 26-30.

9. Pascarella R, Maresca A, Palumbi P, Boriani S. Le complicanze delle fratture laterali del collo del femore trattate con il chiodo gamma (short, standard, long). GIOT. 2002; 28: 181-186

10. Li YH, Zhu D, Li Y, Zhao T, Cao Z, Tan L. Comparison of internal fixation with Gamma3 Long nails and INTERTAN nails in the treatment of Seinsheimer type $\mathrm{V}$ subtrochanteric femoral fractures in elderly patients. Medicine (Baltimore). 2019; 98: e16140.

11. Li F, Sang W, Wang Q, Huang J, Lu H. Subtrochanteric fracture treatment: a retrospective study of 46 patients. Med Princ Pract. 2011; 20: 519-524.

12. Leung $\mathrm{KS}$, Chen $\mathrm{CM}$, So WS, Sato $\mathrm{K}$, Lai $\mathrm{CH}$, Machaisavariya $\mathrm{B}$, et al. Multicenter trial of modified Gamma nail in East Asia. Clin Orthop Relat Res. 1996; 323: 146-154.

13. Pervez $\mathrm{H}$, Parker MJ. Results of the long Gamma nail for complex proximal femoral fractures. Injury. 2001; 32: 704-707.

14. Leung KS, Taglang G, Schnettler R. Practice of Intramedullary Locked Nails New Developments in Techniques and Applications. Berlin, Germany: Springer-Verlag. 2006; 43-56.

15. Krappinger D, Wolf B, Dammerer D, Thaler M, Schwendinger $P$, Lindtner RA. Risk factors for nonunion after intramedullary nailing of subtrochanteric femoral fractures. Arch Orthop Trauma Surg. 2019; 139: 769-777.

16. Sehat K, Baker RP, Pattison G, Price R, Harries WJ, Chesser TJS. The use of the long gamma nail in proximal femoral fractures. Injury. 2005; 36: 13501354.

17. Westacott D, Bould M. Outcome in 36 elderly patients treated with the Gamma3 Long Nail for unstable proximal femoral fracture. Acta Orthop Belg. 2011; 77: 68-72.

18. Edwards SA, Pandit HG, Clarke HJ. The long gamma nail: a DGH experience. Injury. 2000; 31: 701-709.

19. Bjorgul K, Novicoff WM, Saleh KJ. American Society of Anesthesiologist Physical Status score may be used as a comorbidity index in hip fracture surgery. J Arthroplasty. 2010; 25: 134-137.

20. Yoon RS, Donegan DJ, Liporace FA. Reducing subtrochanteric femur fractures: tips and tricks, do's and don'ts. J Orthop Trauma. 2015; 29: S28-S33.

21. Horner NS, Samuelsson K, Solyom J, Bjorgul K, Ayeni OR, Östman B. ImplantRelated Complications and Mortality After Use of Short or Long Gamma Nail for Intertrochanteric and Subtrochanteric Fractures: A Prospective Study with Minimum 13-Year Follow-up. JB JS Open Access. 2017; 2: e0026. 